\title{
A STUDY OF CLINICAL FEATURES AND LABORATORY PROFILE OF DENGUE FEVER IN OUTPATIENT SETTING
}

\author{
Reda Goweda ${ }^{1,3}$ and Ahmed Faisal ${ }^{2}$ \\ ${ }^{1}$ Department of Family Medicine, Faculty of Medicine, Suez Canal University, Egypt. \\ ${ }^{2}$ Department of Infectious and Endemic Diseases, Faculty of Medicine, Suez Canal University, Egypt. \\ ${ }^{3}$ Department of Community medicine, Faculty of Medicine, Umm Al-Qura University, Saudi Arabi.
}

Corresponding author: Reda Abdelmoaty Goweda

Email: redagoweda@yahoo.com

\begin{abstract}
Dengue fever (DF) is one of the common mosquito-borne viral diseases. It is transmitted by Aedes aegypti and Aedes albopictus. The aim to study the clinical and laboratory manifestations of serologically confirmed cases of DF in outpatient clinics. This cross-sectional study was carried out in outpatient setting. According to WHO criteria all patients above 14 years old who were suspected to have DF were tested by IgM dengue antibody test. Additionally all patients underwent history, clinical examination and investigation including complete blood count, liver function test and abdominal ultrasound. Data was analyzed using SPSS version 20.0 ut of 126 patients presented with acute febrile illness, 71 (56.3\%) had seroreactivity for dengue IgM antibodies. Fever, headache and muscloskeletal pain were the most common clinical presentation (100\%). Decreased appetite (92.9\%), retro-orbital pain (78.8\%) and dizziness(64.7\%) were the next common symptoms. 29(40.8\%) had hepatomegally, $11(15.4 \%)$ had splenomegally. Elevated serum alanine aminotransferase (ALT) and aspartate aminotransferase (AST) were observed in 58(81.6\%) and 53(74.6\%) respectively. Lastly; Fever associated with headache, retro-orbital pain, along with thrombocytopenia and elevated liver transaminases should prompt a clinician on the possibility of DF. Increased community awareness and vector control measures need to be strengthened to reduce the burden of dengue cases.
\end{abstract}

Keywords: Dengue fever, Clinical, Laboratory.

\section{INTRODUCTION}

Dengue fever (DF) is an acute febrile illness transmitted by Aedes aegypti and Aedes albopictus and considered one of the most important mosquito-borne viral diseases ${ }^{1}$. The World Health Organization (WHO) classified DF as one of the top 10 threats to global health in 20192. It was estimated that 390 million person infected by dengue virus per year, out of them 96 million can manifest clinically ${ }^{3}$.

In Saudi Arabia, isolation of the dengue virus was done for the first time during an outbreak, this was happened in 1994 in Jeddah, where 289 established cases were reported ${ }^{4}$.The ministry of health in Saudi Arabia recorded 3350 dengue cases in $2009^{5}$. Recent systematic review (2016) found that the studies done inside Saudi Arabia found that the prevalence of DF ranging from $31.7 \%$ to $56.9 \%$ among patients presented with acute febrile illness clinically suggesting dengue fever. Studies were done in Jizan(2013) , Jeddah (2006) and Makkah (2004) found the prevalence of $31.7 \%$ , $48.75 \%$ and $56.9 \%$ respectively ${ }^{7,8}$. DF is endemic only in certain cities in Saudi Arabia (Jeddah, Makkah, Madinah and Jizan), this may be due to high humidity and temperatures that may help worsen the condition ${ }^{10,11}$.

The management of DF is usually asymptomatic by antipyretics and pain killer to alleviate muscle and bone pain. Sever cases may need hospitalization and good hydration ${ }^{1}$.

In 2019, Makkah received 7.5 million Umrah visa holding visitors from different countries worldwide so Makkah's community is different and endemic diseases should be controlled. This study was carried on the authors" place of work (Makkah) which serve the people belong to the university (students ,administration staff and education staff and their families). So most of those people have high education which differs from the general population. In addition, the authors noticed that recording of dengue fever was increased in the last two years and there was monocytosis in most of dengue patients' profile which was not mentioned in the literature .So this study was done to confirm the authors' notices and to determine the patients' characteristics. Accordingly more prevention and control can be applied.

\section{METHODS}

This cross-sectional observational study was carried out in outpatient clinics of Um Alqura University medical Center, Saudi Arabia during period of July 2018 to November 2019. WHO classified dengue into dengue (with / without warning signs) and sever dengue according to the severity of the disease. Dengue can be clinically suspected when a patient presents with a high 
fever $\left(40^{\circ} \mathrm{C} / 104^{\circ} \mathrm{F}\right)$ and has two of the following symptoms at the same time (severe headache, retro-orbital pain, muscular and joint pains, nausea, vomiting, enlarged glands and skin rash) ${ }^{1}$.

Comprehensive sample of all patients $\geq 14$ years old who attended to the clinics complaining of acute febrile illness at least 24 hours and were suspected to have DF according to the mentioned WHO criteria were included in the study without sampling technique during period of July 2018 to November 2019. Patients didn't met WHO criteria and patients with confounding factor such as coinfection, bone marrow diseases that may altered clinical and laboratory results were excluded from study. The target sample was 162 according to the equation $n=z 2 p(1-p) / d 2$. Where $n$ is the sample size, $Z$ is the statistic corresponding to level of confidence (1.96), $P$ is the latest prevalence which was $12.8 \%$ of a study was done in Almadina in Saudi Arabia ${ }^{12}$ and $\mathrm{d}$ was proportion of sampling error $(5 \%)$.

All suspected patients underwent Dengue IgM antibody test depending on enzyme-linked immunosorbent assay (ELISA).

All such patients who were included in the study underwent detailed history, clinical examination and investigation. The history included age, gender, socioeconomic, and history of chronic diseases or medications. Clinical examination included blood pressure, pulse, body temperature, lymph node, skin rash, yellow sclera, abdominal and cardiac examination.

Laboratory investigations done were hemoglobin, total leucocyte count (TLC) and differential leucocyte count (DLC), platelet count, hematocrit (HCT), liver function tests (LFT), blood urea, serum creatinine ,CPK , LDH and CRP .All patients exposed to abdominal ultrasound scan to detect organomegally, ascites, pleural effusion and enlarged lymph nodes . Other relevant investigations were performed according to the clinical conditions of the patients. Informed consent was taken from the patients and data collection and confidentiality were protected to the maximum possible standards.

Data was analyzed using SPSS version 20. Dependent variable was seroreactivity while independent variables were age of the patients, systolic BP, CBC parameter, LFT and CRP Comparison between seropositive and seronegative cases was done by non-parametric $\mathrm{t}$ test and $\mathrm{P}$ - value less than 0.05 was considered significant.

\section{RESULTS}

We recruited 126 patients who attended to the clinics with WHO suspected criteria during period of July 2018 to November 2019. We didn't reach the target sample size (162) because of the low rate due to the clinics received only the people belong to the university (students ,administration staff and education staff and their families). Out of 126 patients presented with acute febrile illness, 71 (56.3\%) had seroreactivity for dengue IgM antibodies. Out of those having DF, 44 (61.9\%) were males and $27(38.1 \%)$ were females with mean of the age was $31.30+9.68$.

On analysis of patients had DF it was found that; Fever, headache and muscloskeletal pain were the most common clinical presentation and it was present in all the patients. Decreased appetite (92.9\%), Retro-orbital pain(78.8\%) and dizziness $(64.7 \%)$ were the next common symptoms. Nausea and vomiting were present in $35(49.2 \%)$ patients. 25(35.2\%) patients had diarrhea. Positive tourniquet test was observed in $7(9.8 \%)$ of the patients while minor bleeding was noticed in $4(5.6 \%)$ of the patients in the form of bleeding per gums and petechiae. No major bleeding in our population was recorded. 19(26.7\%) patients had skin rash. $17(23.9 \%)$ of the patients had low systolic BP $(<90 \mathrm{mmHg})$. 8(11.2\%) had enlarged more than group of lymph nodes. (Table 1 ).

On ultrasound examination it was found that $34(47.8 \%)$ had bight liver texture, 29(40.8\%) had hepatomegally, 11 (15.4\%) had splenomegally , $6(8.4 \%)$ had gall bladder edema and only $1(1.4 \%)$ has pleural effusion. (Table 1 ).

Among haematological parameters, 53 patients (74.6\%) had thrombocytopenia with platelet $<100000 /$ cc while leucopenia (<4000/cc) was noticed in $33.8 \%$. Elevated serum alanine aminotransferase (ALT) and aspartate aminotransferase (AST) were observed in $58(81.6 \%)$ and $53(74.6 \%)$ respectively. $7(9.8 \%)$ of the patients had hyperbilirubinaemia. (Table 2).

On comparing DF patients with seronegative patients it was found that factors associated with seroreactivity; ALT, AST , CRP ,low WBC count , low platelets count, high monocyte count and low neutrophils count with P-values of 0.014 , $0.002,0.020,0.034,0.000,0.012$ and 0.036 respectively. On the hand age, systolic BP, Hemoglobin level, Bilirubin , LDH , Lymphocytic count, Esonphilic count and basophilic count didn't show significant analysis. (Table 3) 
Table 1: Clinical features of patients with dengue fever

\begin{tabular}{lc}
\hline Symptoms and Clinical signs & $\mathbf{N}(\%)$ \\
\hline Fever & $71(100 \%)$ \\
Headache & $71(100 \%)$ \\
Abdominal pain & $23(32.3 \%)$ \\
Bone ache & $71(100 \%)$ \\
Muscle and joint pain & $71(100 \%)$ \\
Retro-orbital pain & $56(78.8 \%)$ \\
Blurring of vision & $6(8.4 \%)$ \\
Decreased appetite & $66(92.9 \%)$ \\
Nausea and or Vomiting & $35(49.2 \%)$ \\
Diarrhea & $25(35.2 \%)$ \\
Cough & $10(14 \%)$ \\
Skin Rashes & $19(26.7 \%)$ \\
Positive tourniquet test & $7(9.8 \%)$ \\
Dizziness & $46(64.7 \%)$ \\
Bleeding & $4(5.6 \%)$ \\
Lymphadenopathy & $8(11.2 \%)$ \\
Hypotension & $17(23.9 \%)$ \\
Ultrasonography findings & \\
Splenomegally & $11(15.4 \%)$ \\
Hepatomegally & $29(40.8 \%)$ \\
Bright liver texture & $34(47.8 \%)$ \\
Gall bladder edema & $6(8.4 \%)$ \\
Pleural effusion & $1(1.4 \%)$ \\
\hline
\end{tabular}

Table 2: Laboratory parameters of dengue fever patients

\begin{tabular}{lc}
\hline \multicolumn{1}{c}{ Laboratory results } & N (\%) total 71 \\
\hline TLC $(<4000 / \mathrm{cc})$ & $24(33.8 \%)$ \\
Platelets $(<100000 / \mathrm{cc})$ & $53(74.6 \%)$ \\
AST $(>40 I U / L)$ & $58(81.6 \%)$ \\
ALT $(>45 I U / L)$ & $53(74.6 \%)$ \\
Total bilirubin $(>1.2 \mathrm{mg} / \mathrm{dl})$ & $7(9.8 \%)$ \\
\hline
\end{tabular}

Table 3: Comparison between seropositive with seronegative dengue IgM antibodies

\begin{tabular}{|c|c|c|c|c|c|}
\hline & \multicolumn{2}{|c|}{$\begin{array}{l}\text { Positive dengue } \lg M \\
\text { antibodies }\end{array}$} & \multicolumn{2}{|c|}{$\begin{array}{l}\text { Negative dengue } \lg M \\
\text { antibodies }\end{array}$} & \multirow[t]{2}{*}{$\mathrm{p}$} \\
\hline & Mean & SD & Mean & ?SD & \\
\hline Age & 31.30 & 9.68 & 32.94 & 11.85 & 0.396 \\
\hline systolic & 111.40 & 10.15 & 109.24 & 12.34 & 0.291 \\
\hline Hemoglobin(gm/dl) & 13.44 & 2.08 & 13.59 & 1.98 & 0.688 \\
\hline $\operatorname{ALT}(\mathrm{IU} / \mathrm{ml})$ & 83.47 & 56.27 & 57.09 & 61.68 & 0.014 \\
\hline AST(IU/ml) & 96.81 & 51.51 & 61.12 & 76.00 & 0.002 \\
\hline Bilirubin(total)(mg/dl) & 0.88 & 0.33 & 0.79 & 0.29 & 0.122 \\
\hline CRP & 25.35 & 20.03 & 18.03 & 14.39 & 0.020 \\
\hline LDH & 418.60 & 243.60 & 350.35 & 234.22 & 0.119 \\
\hline WBC & 3.65 & 1.48 & 4.17 & 1.17 & 0.034 \\
\hline Neutorophils & 41.71 & 13.71 & 46.90 & 13.52 & 0.036 \\
\hline Lymphocytes & 45.12 & 12.16 & 42.69 & 12.52 & 0.274 \\
\hline Monocytes & 8.64 & 5.15 & 6.50 & 4.08 & 0.012 \\
\hline Esonphils & 1.92 & 1.83 & 1.55 & 1.12280 & 0.195 \\
\hline Basophils & 2.45 & 2.19 & 2.59 & 2.06 & 0.711 \\
\hline Platelet count $(/ \mathrm{cmm})$ & 135.09 & 56.95 & 186.36 & 63.20 & 0.000 \\
\hline
\end{tabular}




\section{DISCUSSION}

In the current study out of 126 presenting with acute febrile illness, 71 (56.3\%) showed seroreactivity for dengue IgM antibodies which is in unison with other similar studies in Makkah and Jeddah, Saudi Arabia that reported a seroprevalence of $56.25 \%$ and $48.75 \%$ respectively ${ }^{7.8}$. Our study result was slightly higher than results observed by study done in Aseer and Jizan, Saudi Arabia which reported seropositivity in $31.7 \%$ of the suspected cases $^{8}$. The higher prevalence in our study because the disease is endemic in Makkah due to its atmosphere and nature.

The current study has shown that DF is common in male gender and young age this may be due to males are more exposed to the virus because most of females in Makkah wear cloths and Nikab which cover the hole body including face. These findings are consistent with other studies ${ }^{13,16}$. Without statistically significant relationships studies performed in Australia, France and Mexico, reported that DF was more common among female gender ${ }^{17,19}$.

Headache, myalgia and retro-orbital pain are well-known symptoms of DF. As compared to similar studies ${ }^{13,14,20,21}$, the main symptoms and clinical signs of our patients were headache, bone ache, myalgia, joint pain and retro-orbital pain.

All of our patients in the current study experienced headache. Most of the previous studies describe similar results, however lower incidence of headache $(9 \%)$ was reported by Awasthi et al. ${ }^{22}$ which is much less than our study patients. Myalgia was found in $100 \%$ of our patients which is comparable with previous studies $^{23}$, however study conducted by Mohamed et al., showed lower incidence (32.87\%) (24). Retro-orbital pain was noticed in $78.8 \%$ of the patients in present study comparable to Deshwal et al., (18.3\%) and Lepakshi et al., (14\%) ${ }^{25.26}$; however study done by Nandini Chatterjee et al., had $90 \% 21$.

In our study, abdominal pain was found in $32 \%$ of the patients which correlates with the previous studies, however studies conducted by Ragini Singh et al., and Munde et al., showed slightly lower incidence of $3.6 \%$ and $15 \%$ respectively ${ }^{27,28}$. In our study, $49.2 \%$ of the patients presented with nausea/vomiting comparable to $25 \%$ in study conducted by Munde et al., however Ragini Singh et al., reported only $11.4 \% 28$.

Skin rash was noticed in $26.7 \%$ of our patients, this was comparable to the prevalence of skin rash (26\%) documented by Karoli et al. ${ }^{29}$. This presentation is less in other studies by ltoda et al. in japan , and Rahim et al. in a Bangladesh, showed higher incidence $(82 \%, 78.5 \%$ respectively $)^{30,31}$.
Hemorrhagic manifestation is one of the complications of DF due to low platelet count and increased capillaries permeability of blood vessels. Bleeding manifestations and a positive tourniquet test were relatively uncommon in our study. These findings are inconsistent with three studies performed in Saudi Arabia ${ }^{13,14,20}$. Bleeding was seen in $5.43 \%$ of cases and positive tourniquet test was recorded in $16.50 \%$ of patients in a study by Rajesh Deshwal et al. ${ }^{25}$, however other study recorded a higher incidence $(40 \%)^{28}$.

Abdominal ultrasound scan has an important role in DF for diagnosis of organomegally, ascites, gall bladder edema and pleural effusion. Only one patient among this study population, presented to us with pleural effusion but no patient with ascites. In a study by Mandal et al. ${ }^{32}$ ascites was present in $8.1 \%$ and pleural effusion in $18.9 \%$ of cases. In present study we found that $8.4 \%$ of the patients had gall bladder edema, it could be due to direct viral effect on the gall bladder wall leading to increased vascular permeability and edema. Hepatomegally was detected in $40.8 \%$ of our cases while Deshwal, $\mathrm{R}$ et al., ${ }^{25}$ reported $14.8 \%$. the high incidence in our study may be due to pre existing fatty liver due to sedentary life and obesity. In the present study $15.4 \%$ of patients had splenomegally, this is consistent with Deshwal, R et al. ${ }^{25} 13.2 \%$.

The main hematological abnormalities were thrombocytopenia, low WBC count and elevated liver enzymes which are similar to previous studies in Saudi Arabia ${ }^{7,9,20}$.

Thrombocytopenia $(<100000 / \mathrm{cc})$ documented in $74.6 \%$ of all dengue cases in our study. Rashmi et al. ${ }^{33}$ have documented thrombocytopenia $(<140000 / \mathrm{cc})$ in all cases, but platelet count less than 100000 were reported in $89.4 \%$ cases. Mandal et al. ${ }^{32}$, in their series of patients have shown platelet count $(<50000 / \mathrm{cc})$ in $37.8 \%$. A study conducted in 2014 found the common laboratory presentations of DF were thrombocytopenia $(96 \%)^{35}$.

Among the laboratory profile, leukopenia $(<4000 / \mathrm{cmm})$ was noticed in $33.8 \%$ of our patients. A study by Itoda ${ }^{33}$ found that low WBC count was observed in $71 \%$ of cases. However studies done by Munde et al.,(35) and Karoli ${ }^{29}$ reported leukopenia in $50 \%$ and $89 \%$ of their patients respectively. Ageep $^{36}$ detected leucopenia in $90 \%$ of cases while Mandal ${ }^{32}$ found leucopenia in $29.73 \%$ of cases.

Liver involvement is common in DF and its damage manifests as elevation of ALT and AST, hypoalbuminaemia, and prolonged of PT and PTT37.Elevated liver transaminases were noted in the majority of the cases in this study $(82 \%)$.In study by Deshwal ${ }^{25}(88.54 \%)$ patients showed elevated ALT and AST . Kularatne et al documented elevated transaminases in $88 \%$ of 
cases. Another study recorded that $57 \%$ of patients had elevated ALT and $49 \%$ had raised $\mathrm{AST}^{22}$. Previous studies in saudi Arabia reported similar results ${ }^{7}$.

In our study there was significant difference in monocyte count being high in DF group. Additionally no case mortality was reported among our sample population, however previous studies observed mortality rate of $4 \%^{38,39}$.

\section{LIMITATION OF THE STUDY}

This study is a single center outpatient clinic based study recruited small sample size. This small sample during this long time because of the low rate due to the clinics received only the people belong to the university (students ,administration staff and education staff and their families) Also, there was lacking of information regarding if the infection is primary or secondary as well as dengue serotype. A multi- center and tertiary hospitals cross sectional study with larger sample size that include all general population would prove more useful conclusions.

\section{CONCLUSION}

Dengue is a challenging infectious disease with a major public health concern that can present with no symptoms to sever life threatening symptoms. Fever associated with headache, retro-orbital pain,thrombocytopenia and elevated liver transaminases show high susceptibility criteria for dengue diagnosis. Monocytosis was observed in our study in dengue fever group and further studies are needed to confirm this finding. Community awareness regarding prevention and control and mode of transmission more is needed .Additionally , continuous surveillance and mosquito control measures in Makkah are needed.

CONFLICT OF INTEREST: NO

FUNDING: No

\section{REFERENCES}

1. World Health Organization. Media center: Dengue and Severe Dengue. http://www.who.int/media centre/factsheets/fs117/en/

2. Ten threats to global health in 2019. World Health Organization. Available at https://www.who.int/emergencie s/ten-threats-to-global-health-in2019. 2019; Accessed: Feb 2, 2019.

3. Bhatt S, Gething PW, Brady OJ, et al., The global distribution and burden of dengue.Nature. 2013 Apr25;496(7446 ):504-7.
4. Fakeeh M, Zaki AM. Virologic and serologic surveillance for dengue fever in Jeddah, Saudi Arabia, 19941999.Am J Trop Med Hyg, 65 (2001), pp. 764-767.

5. Ministry of Health, Department of StatisticsHealth statistical year book 2009 Saudi Ministry of Health, Riyadh, KSA (2010) ISSN: 1319-3228.

6. Alhaeli A, Bahkali S, Ali A, Househ MS, ElMetwallyAA.The epidemiology of Dengue fever in Saudi Arabia: A systematic review. J Infect Public Health. 2016 Mar-Apr;9(2):117-24.

7. Ayyub M, Khazindar AM, Lubbad EH, Barlas S, Alfi AY, Al-Ukayli S.Characteristics of dengue fever in a large public hospital, Jeddah, Saudi Arabia.J Ayub Med Coll Abbottabad, 18 (2006), pp. 9-13.

8. Al-Azraqi TA, El Mekki AA, Mahfouz AA.Seroprevalence of dengue virus infection in Aseer and Jizan regions, Southwestern Saudi Arabia. Trans R Soc Trop Med Hyg. 2013 Jun;107(6):368-71.

9. Khan NA, Azhar El, El-Fiky S, et al.Clinical profile and outcome of hospitalized patients during first outbreak of dengue in Makkah, Saudi Arabia. Acta Trop. 2008 Jan;105(1):39-44.

10. Khormi HM, Kumar L. Assessing the risk for dengue fever based on socioeconomic and environmental variables in a geographical information system. Geospat Health. 2012 May;6(2):171-6.

11. Khormi HM, Kumar L. The importance of appropriate tem-poral and spatial scales for dengue fever control andmanagement. Sci Total Environ 2012;430:144-9.

12. A.A. ElBadry, H.A. ElBeshbishy, K.H. Al-Ali, A.M. Al-Hejin, W.S.M. El Sayed. Molecular and seroprevalence of imported dengue virus infection in Al-Madinah, Saudi Arabia. Comp Clin Pathol, 23 (4) (2014), pp. 861-868.

13. Srikiatkhachorn A, Gibbons RV, Green S, Libraty DH, Thomas SJ, et al. Dengue hemorrhagic fever: the sensitivity and specificity of the world health organization definition for identification of severe cases of 
dengue in Thailand, 1994-2005. Clin Infect Dis 2010; 50:1135-1143.

14. Mohan D K, Shiddappa, Dhananjaya M. A Study of Clinical Profile of Dengue Fever in a Tertiary Care Teaching Hospital. Sch J App Med Sci 2013; $1: 280-282$.

15. Agarwal R, Kapoor S, Nagar R, Misra A, Tandon R, Mathur A, et al. A clinical study of the patients with dengue hemorrhagic fever during the epidemic of 1996 at Lucknow, India. Southeast Asian J Trop Med Public Health 1999;30:735-40.

16. Alzahrani AG, Al Mazroa MA, Alrabeah AM, Ibrahim AM,Mokdad AH, Memish ZA. Geographical distribution and spatio-temporal patterns of dengue cases in Jeddah Governorate from 2006-2008. Trans R Soc Trop Med Hyg2013;107:23-9.

17. Horta MA, Bruniera R, Ker F, Catita C, Ferreira AP. Temporal relationship between environmental factors and the occurrence of dengue fever. Int J Environ Health Res 2014:1-11.

18. García-Gutiérrez MD, Romero-Zepeda $\mathrm{H}$, Salvador Romero-Márquez R. Risk factors associated to the epidemic outbreak of dengue virus infection. Rev Med Inst Mex Seguro Soc2013;51:628-34.

19. Raza FA, Rehman SU, Khalid R, Ahmad $\mathrm{J}$, Ashraf S, lqbalM, et al. Demographic and clinicoepidemiological features of dengue Fever in Faisalabad, Pakistan. PLOS ONE2014;9:e89868.

20. Ahmed MM. Clinical profile of dengue fever infection in KingAbdul Aziz University Hospital Saudi Arabia. J Infect DevCountries 2010;4:503-10.

21. Nandini, C., Mainak, M., Sinjon, G., Manas, M., Chiranjib D, Kartik P. An observational study of dengue fever in a tertiary care hospital of Eastern India. J. Assoc. Physicians India, 62: 12-5.

22. Awasthi S., Singh V., Kumar S., Kumar A., and Dutta S. The changing clinical spectrum of Dengue fever in the 2009 epidemic in north India: a tertiary teaching hospital based study," Journal of Clinical and Diagnostic Research,vol. 6, no. 6,pp. 999-1002, 2012.
23. Horvath, R., Mcbride WJH, Hanna JN. Clinical features of hospitalized patients during dengue 3 epidemic in Far North Queensland 1997-99. Dengue Bulletin. 1999; 23:24- 29.

24. Mohamed K,Kalavathi $\mathrm{P}$, Mehul R, Karthik M,Asfiya A,Kumaraswamy R. A study of clinical and laboratory profile of dengue fever in tertiary care hospital in central Karnataka, India. Glob. J. Med. Res. B: Pharma Drug Discov. Toxicol. Med., 14(5).

25. Deshwal, R., Qureshi, M.I., Singh, R. Clinical and Laboratory Profile of Dengue Fever. J Assoc Physicians India. 2015 Dec;63(12):30-32.

26. Lepakshi, G., N. Padmaja, Rafiq Pasha, H. A study of clinical profile of Adult patients with dengue fever. Indian J. Appl. Res., 2015,5:820-823.

27. Munde, D, Shetkar U. Clinical Features and Haematological Profile of Dengue Fever. Indian Journal of Applied Research. 2013; 3:131-132.

28. Ragini S, Singh P., Niaz A. A Study of Clinical and Laboratory profile of dengue fever in a tertiary care centre of Uttarakhand, India. Int. J. Res Med Sci. 2014; 2:160-163.

29. Karoli R, Fatima J, Siddiqi Z, Kazmi KI, Sultania AR.Clinical profile of dengue infection at a teaching hospital in North India. J Infect Dev Ctries. 2012 Jul 23;6(7):551-4.

30. Itoda, I., Masuda, G, Suganuma A et al. Clinical features of 62 importedcases of dengue fever in Japan. Am J Trop Med Hyg 2006; 75 (3): 470-4.

31. Rahim, M.A., Sikder MS. Clinicopathologic manifestations and outcome of dengue fever and dengue haemorrhagic fever. Bangladesh Med Res Counc Bull. 2005 Apr;31(1):36-45.

32. Mandal SK, Ganguly J, Koelina Sil et al.Clinical profiles of dengue fever in a teaching hospital of eastern india. Nat J Med Res 2013; 3:173-176.

33. Rashmi KS, Jagdeesh, Ravikumar KL, Giridhar UP, Arun KR. Serological markers prevalence and trend of probable dengue infection at a tertiary care hospital in Banglore. J Evolution of Medical and Dental Sciences. 2013;2(36):6968-77. 
34. Mena Lora AJ, Fernandez J, Morales A, Soto Y, Feris-Iglesias J, Brito MO. Disease severity and mortality caused by dengue in a Dominican pediatric population. Am J Trop Med Hyg. 2014 Jan;90(1):169-72.

35. Munde, D.D., Shetkar U B. Clinical Features and Haematological Profile of Dengue Fever. Indian Journal of Applied Research. 2013; 3:131-132.

36. Ageep AK, Malik AA, Elkarsani MS. Clinical presentations and laboratory findings in suspected cases of dengue virus. Saudi Med J. 2006 Nov;27(11):1711-3.

37. De Macedo FC, Nicol AF, Cooper LD, Yearsley M, Pires AR, Nuovo GJ. Histologic, viral, and molecular correlates of dengue fever infection of the liver using highly sensitive immunohistochemistry. Diagn Mol Pathol. 2006 Dec. 15(4):223-8.

38. Chatterjee $N$, Mukhopadhyay $M$, Ghosh S, Mondol M, Das C, Patar K. An observational study of dengue fever in a tertiary care hospital of Eastern India. J Associ Physicians of India. 2014;62(3):224-7.

39. Chhotala YH, Suva CM. A study of clinical profile of dengue fever in a tertiary care hospital of Jamnagar, Gujarat, India. Int J Res Med Sci. 2016;4(10):4500-4. 